\title{
Primary angiitis of the CNS with pure spinal cord involvement: a case report
}

\author{
Chantima Goertz $\cdot$ Christiane Wegner · \\ Wolfgang Brück · Peter Berlit
}

Received: 18 October 2009/Accepted: 23 May 2010/Published online: 18 June 2010

(C) Springer-Verlag 2010

\section{Dear Sirs,}

We report the rare case of a primary angiitis of the CNS (PACNS) with isolated spinal cord manifestation. This 44year-old female presented diffuse dysesthesia with moderate progressive weakness of the lower limbs. There were no cerebral symptoms; cMRI was normal. The diagnosis of PACNS was made by a spinal cord biopsy demonstrating granulomatous vasculitis. The patient was successfully treated with steroids and methotrexate. During a 2 year follow-up our patient did not show any brain or other organ involvement.

The 44-year-old female presented with moderate progressive weakness of both legs and burning sensory symptoms of all limbs and the trunk. In addition to moderate paraparesis, the physical examination revealed a moderate pallhypesthesia of the legs with reduced vibratory sense and a diffuse hyp- and dysesthesia below the level of CD5. The family history was unremarkable for neurological diseases.

The MRI of the cervical spine showed a gadoliniumenhancing lesion in the posterior part of the myelon with intramedullary edema. This lesion extended from cervical vertebral bodies 2-7 (Fig. 1a).

The cerebral MRI and visual evoked potentials were entirely normal, the examination of the CSF revealed a slight pleocytosis $\left(12\right.$ cells $\left./ \mathrm{mm}^{3}\right)$, cytological examination

C. Goertz $(\bowtie) \cdot$ P. Berlit

Alfried Krupp Hospital, Essen, Germany

e-mail: chanti81@arcor.de

C. Wegner · W. Brück

University Hospital Göttingen, Göttingen, Germany showed regular cells. Oligoclonal bands were absent which makes multiple sclerosis highly improbable.

Laboratory examinations did not reveal any evidence for either metabolic (vitamin-B12, folate), autoimmune or infectious disorder. We found no aquaporin-4-antibodies. With normal VEP, we had no evidence for neuromyelitis optica.

We started with intravenous high dose methylprednisolone therapy ( $1 \mathrm{~g}$ for 5 days), but symptoms relapsed after 3 months of disease freedom.

Before introduction of another immunosuppressive therapy, and to exclude a spinal lymphoma, a biopsy of the spinal lesion was taken. The neuropathological examination revealed a granulomatous vasculitis with transmural inflammation consisting of lymphocytes, epitheloid cells and giant cells (Fig. 2a-h). There was no evidence for systemic organ involvement assessed by thoracic and abdominal computer tomography.

Treatment was started with methotrexate $(20 \mathrm{mg} /$ week) combined with prednisolone (tapered from 80 to $10 \mathrm{mg} / \mathrm{d}$ within 6 weeks).

Neurological examination at 3 month intervals revealed normal findings except a slight dysesthesia in the legs.

A follow-up MRI after 24 months of treatment demonstrated an obvious regression of the signal intensities within the cervical cord (Fig. 1b), whereas the cMRI remained normal.

Spinal symptoms are a rare presentation of primary CNS vasculitis [1]. The previously reported cases with spinal cord involvement also showed progressive paraparesis and dysesthesia as the most common clinical symptoms [2]. However, usually these symptoms occur in the course of the disease and not at disease onset as in our case. A review of the literature showed only eight cases with initially pure spinal cord involvement [3]; half of these cases developed brain lesions later on $[1,4]$. 


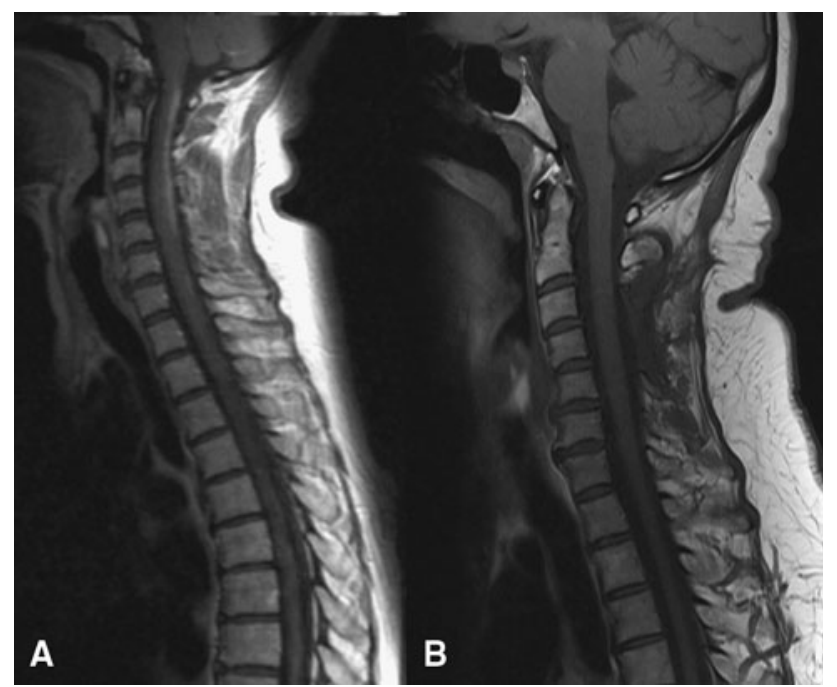

Fig. 1 a MR study of the untreated patient. Sagittal FSE T1-weighted image shows a big, homogeneously enhancing lesion located in the cervical cord. b Follow-up MR study 24 months later. Sagittal FSE T1-weighted image shows no evidence of contrast enhancement of the cervical cord
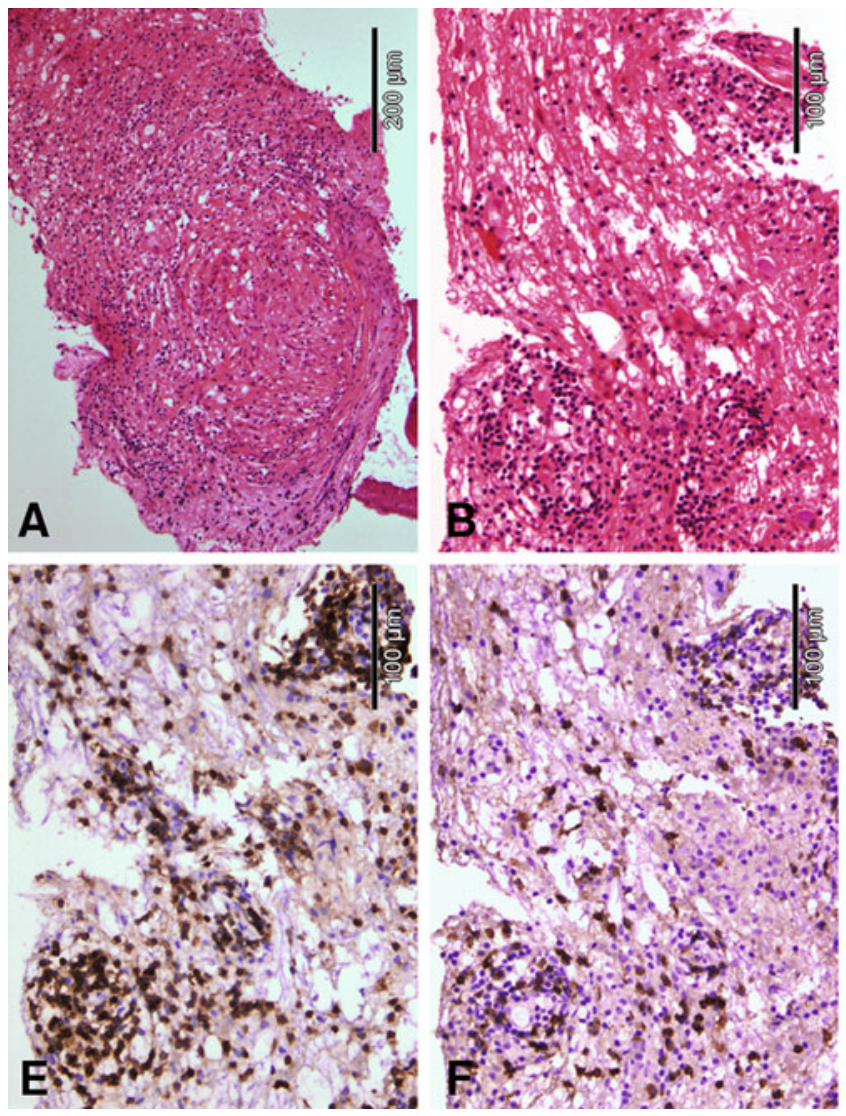

Fig. 2 The histological features of primary spinal vasculitis. The neuropathological examination revealed vascular thrombosis (a $100 \times$ magnification, H\&E staining) and vessel walls with transmural infiltration mainly consisting of several lymphocytes (b 200× magnification, $H \& E$ staining). In addition, there were epitheloid cells and giant cells (c 400× magnification, H\&E staining) which are characteristic for a granulomatous vasculitis. There was no evidence for demyelination (d 200× magnification, LFB-PAS staining). Immunostainings indicated
The stereotactic biopsy remains the gold standard to assure the diagnosis of a CNS vasculitis [5], but the diagnosis in daily clinical routine is often only based on clinical and imaging findings as well as the response to treatment. This might be a potential reason for overdiagnosing PACNS in clinical routine. As illustrated by our case, histological verification is crucial for the diagnosis.

The standard therapy for PACNS consists of corticosteroids ( $1 \mathrm{mg} / \mathrm{kg}$ prednisolone) and pulsed cyclophosphamide $(0.5 \mathrm{mg} / \mathrm{kg})$. The treatment with cyclophospha mide can have severe side effects [6]. Monotherapy with steroids leads to higher recurrence rates [7]. Since methotrexate has been successfully used in systemic vasculitis [8], we started treatment with a combination of steroids and methotrexate. Our patient reached remission within 3 months and remains stable now more than 2 years later. In biopsy-proven PACNS of the spinal cord, methotrexate seems to be an effective and less toxic alternative to cyclophosphamide.

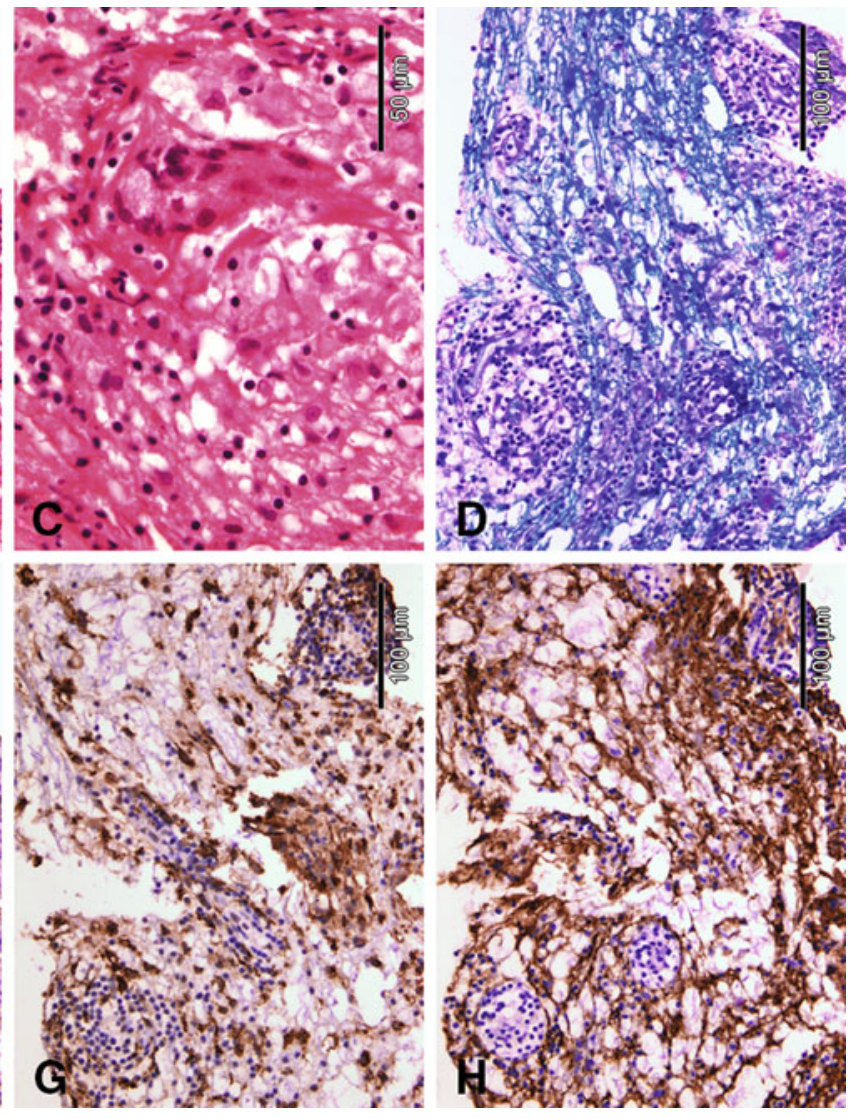

that the vessel walls were mainly infiltrated by CD3-positive T cells (e 200 × magnification, immunohistochemistry for CD3) that were partly CD8-positive cytotoxic $\mathrm{T}$ cells (f $200 \times$ magnification, immunohistochemistry for CD8). Furthermore, several CD68-positive macrophages and activated microglial cells were detectable (g 200× magnification, immunohistochemistry for CD68). Within the CNS tissue, many reactive astrocytes could be observed (h $200 \times$ magnification, immunohistochemistry for glial fibrillic acidic protein) 


\section{References}

1. Salvarani C, Brown RD Jr, Calamia KT, Christianson TJ, Huston J III, Meschia JF, Giannini C, Miller DV, Hunder GG (2008) Primary CNS vasculitis with spinal cord involvement. Neurology 70:2394-2400

2. Campi A, Benndorf G, Martinelli V, Terreni MR, Scotti G (2001) Spinal cord involvement in primary angiitis of the central nervous system: a report of two cases. AJNR Am J Neuroradiol 22:577-582

3. Yoong MF, Blumbergs PC, North JB (1993) Primary (granulomatous) angiitis of the central nervous system with multiple aneurysms of spinal arteries. Case report. J Neurosurg 79:603-607
4. Yuen RW, Johnson PC (1996) Primary angiitis of the central nervous system associated with Hodgkin's disease. Arch Pathol Lab Med 120:573-576

5. Kim JY, Ahn KJ, Jung JI, Jung SL, Kim BS, Hahn ST (2007) Imaging findings of central nervous system vasculitis associated with Goodpasture's Syndrome: a case report. Korean J Radiol 8:545-547

6. Anderson D, Bishop JB, Garner RC, Ostrosky-Wegman P, Selby PB (1995) Cyclophosphamide: review of its mutagenicity for an assessment of potential germ cell risks. Mutat Res 330:115-181

7. Moore PM (2000) Vasculitis of the central nervous system. Curr Rheumatol Rep 2:376-382

8. Yazici Y (2008) Systemic vasculitis treatment and monitoring update, 2008. Bull NYU Hosp Jt Dis 66:228-230 